\title{
Analysis of Handoff Interference and Outage along Arbitrary Trajectories in Cellular Networks
}

\author{
Alexe E. Leu, Member, IEEE, Brian L. Mark, Member, IEEE, and Shensheng Tang, Senior Member, IEEE
}

\begin{abstract}
We introduce a new system performance measure due to handoff called handoff interference, which characterizes the additional interference noise induced by the handoff process. The handoff interference experienced by a mobile unit is determined by the parameters of the handoff algorithm. We present an exact analysis of the handoff interference and the outage probability and develop a discrete-time method to efficiently and accurately compute these performance metrics along arbitrary trajectories in a cellular network. Our numerical results reveal critical tradeoffs among the critical handoff performance metrics, which should be taken into account in dimensioning the handoff parameters to optimize system performance. We discuss an example handoff design problem, which illustrates how to dimension the handoff parameters to optimize this tradeoff using the proposed handoff analysis.
\end{abstract}

Index Terms-Cellular systems, communication system performance, communication system design, handoff algorithms, system outage, radio propagation, discrete-time systems.

\section{INTRODUCTION}

$\mathbf{T}$ HIRD and fourth generation wireless networks are being designed to provide higher bandwidth and more seamless connectivity to mobile users [2]-[4]. Handoffs occur whenever a mobile station moves from the coverage area of one access point or base station, to another. The most common handoff scenario is a mobile station crossing the boundary between two neighboring cells in a cellular system. As wireless networks become more heterogeneous, handoffs between different types of networks will become more prevalent. For example, a cell phone user might take advantage of a local IEEE 802.11 access point for a VoIP call, file transfer, and video-conferencing [5][7]. When the user moves out of range of the access point, a handoff to a $3 \mathrm{G}$ cellular system might be necessary to complete the transaction seamlessly. The handoff behavior of mobile users in such networks has a critical impact on the overall system performance. However, the effect of handoff on system capacity is not as well understood as other aspects of cellular systems, such as equalization, modulation, and coding.

Manuscript received April 10, 2007; revised July 27, 2007; accepted September 16, 2007. The associate editor coordinating the review of this paper and approving it for publication is P. Fan. This work was supported in part by the U.S. National Science Foundation under Grant 0133390 and by a grant from Northrop Grumman Mission Systems. An early version of this work was presented in part at the IEEE Wireless Communications and Networking Conference (WCNC), May 2004 [1].

A. E. Leu is with Shared Spectrum Company, Vienna, VA 22182 (e-mail: aleu@sharedspectrum.com).

B. L. Mark and S. Tang are with the Dept. of Electrical and Computer Engineering, The Volgenau School of Information Technology \& Engineering, George Mason University, Fairfax, VA 22030 (e-mail: \{bmark, stang1\}@gmu.edu).

Digital Object Identifier 10.1109/TWC.2008.070384.
Most studies of handoff performance deal with simplified scenarios, which may not fully characterize the overall performance of the network [8]-[11]. Handoff performance is typically quantified in terms of assignment probability and handoff probability at each point along a trajectory taken by a given mobile station (MS). The assignment probability to a base station $\mathrm{BS}_{i}$ at time $k$, denoted by $P_{i}[k]$, is the probability that an MS moving along a given trajectory is assigned to $\mathrm{BS}_{i}$ at time $k$. The handoff probability from $\mathrm{BS}_{i}$ to $\mathrm{BS}_{j}$ at time $k$, denoted by $P_{i j}[k]$, is the probability that the MS will be handed off from $\mathrm{BS}_{i}$ to $\mathrm{BS}_{j}$ at time $k$. We remark that the handoff and assignment probabilities depend on the particular trajectory taken by the MS. The authors in [8] present different aspects of handoff and discuss handoffrelated features of cellular systems. In [9], an approximate model is developed for evaluating the performance of handoff algorithms based on relative signal strength measurements. This model was extended in [10] by taking into account the absolute signal strength from the current base station (BS) to reduce unnecessary handoffs. In [11], a discrete-time approach is introduced to analyze the handoff performance based on processed relative signal strength measurements. In contrast to the results in [9], [10], exact analytical expressions are derived for the handoff probabilities, which do not involve approximations.

Handoff performance is typically analyzed for a MS moving at constant speed along the canonical straight-line trajectory connecting one base station to another. Handoff performance along such a trajectory is often quantified in terms of metrics such as the mean number of handoffs and the crossover point [9]-[11]. The crossover point is defined as the point along the straight-line trajectory between two base stations, say from $\mathrm{BS}_{i}$ to $\mathrm{BS}_{j}$, at which the probability of assignment to $\mathrm{BS}_{i}$ drops below 0.5. Ideally, the crossover point should be located halfway between $\mathrm{BS}_{i}$ and $\mathrm{BS}_{j}$. However, the ideal situation can only be achieved when no hysteresis or averaging is used in the handoff algorithm. But hysteresis and averaging are necessary to avoid the ping-pong effect in handoff. Thus, the crossover point is a measure of the cell coverage area or the handoff delay incurred by practical handoff algorithms with hysteresis [12], [13]. In dimensioning the handoff algorithm, a tradeoff exists between the mean number of handoffs and the crossover point: a smaller mean number of handoffs corresponds to a larger crossover point. This tradeoff factors into the proper dimensioning of the handoff algorithm.

In practice, an MS may move along an arbitrary trajectory within the coverage area of the network. To characterize the overall performance of the network, it is important to 
consider more basic handoff metrics that pertain to arbitrary mobile trajectories. For example, handoff and assignment probabilities may be defined at each handoff decision instant along any given trajectory. The mean number of handoffs and crossover point along any given trajectory can be derived from the handoff probabilities and assignment probabilities, respectively.

The outage probability, i.e., the fraction of time that the received power from current $\mathrm{BS}$ falls below the required threshold, is another important performance metric that can be defined at each handoff decision instant [14]-[16]. In [14], approximations for the outage probabilities of both hard and soft handoff algorithms are derived through fade margins and the reverse link capacity is investigated through relative interference. In [15], [16], approximations for the outage probability and fade margins are derived based on a model for hard handoff with hysteresis in a two-cell system. The studies of outage behavior in [14]-[16] consider the outage probability only at the stationary midpoint of the canonical straight-line trajectory, which corresponds to a point on an imaginary ideal cell boundary. However, in reality, the midpoint usually does not correspond to the crossover point. Identifying the midpoint with the crossover point leads to an inaccurate assessment of outage behavior.

In this paper, we introduce a new system performance measure called handoff interference, which characterizes at each handoff decision instant along an arbitrary trajectory, the additional interference noise induced by the handoff process. Previous works related to handoff have ignored the impact of the interference induced by the handoff process. By introducing handoff interference, the important tradeoff between seamless connectivity (i.e., number of handoffs) and the interference associated with handoff can be taken into account. Using the discrete-time approach for handoff analysis discussed in [11], [17], we develop an exact method to compute the handoff interference along arbitrary trajectories. We also derive exact expressions for computing the outage probabilities along an arbitrary trajectory, in contrast to [14][16]. Our numerical results reveal critical tradeoffs among the obtained performance metrics, which should be taken into account in dimensioning the handoff parameters to optimize system performance.

To characterize handoff performance for arbitrary trajectories in a cellular network, we approximate a general path by a piecewise linear path within a reduced geometric structure derived from the cellular network geometry. In this way, we can obtain a concise characterization of handoff performance over a wide range of mobile trajectories in the network. This characterization provides a measure of the overall signaling load incurred by the handoff algorithm.

The remainder of the paper is organized as follows. Section II describes the basic system model and results from the discrete-time hard handoff analysis of [11]. Section III introduces the handoff interference metric and develops a numerical procedure for computing this metric. Section IV develops an analysis of outage probabilities along a given trajectory. Section V introduces a methodology for characterizing handoff performance over a wide range of mobile trajectories in a cellular network. Section VI presents numerical results of handoff performance obtained using our approach and discusses the application of handoff interference and outage analysis to a handoff design problem. Finally, the paper is concluded in Section VII.

\section{System Model AND DiscRete-TIME HANDOFF ANALYSIS}

\section{A. Signal Strength Model}

The cellular network consists of a set, $\mathcal{C}$, of base stations deployed in a coverage area. The MS makes handoff decisions based on measurements of the pilot signal strength received from the two nearest base stations, $\mathrm{BS}_{i}$ and $\mathrm{BS}_{j}$. Our analysis of handoff performance is based on a discrete-time model for the signal strength measurements. In the discrete-time model, the mobile unit samples the pilot signal strengths at time instants $t_{k}=k t_{s}$, where $t_{s}$ is the sampling interval. The distance between successive sampling positions of the mobile is denoted by $d_{s}=v t_{s}$. The discrete-time model captures the handoff behavior of an MS more accurately than its continuous-time counterpart since all signal strength measurements are sampled.

In discrete-time, the measured pilot signal strength $Y_{i}[k]$ from base station $\mathrm{BS}_{i}$ under the standard lognormal fading model can be expressed in $\mathrm{dB}$ as [11], [18]:

$$
Y_{i}[k]=m_{i}[k]+W_{i}[k]+Z_{i}[k],
$$

where $m_{i}[k], W_{i}[k]$, and $Z_{i}[k]$ represent the path loss, shadowing, and fast fading components, respectively ${ }^{1}$. The path loss component $m_{i}[k]$ is given by

$$
m_{i}[k]=\kappa_{1}-\kappa_{2} \log \left(\left\|\mathbf{r}\left(t_{k}\right)-\mathbf{b}_{i}\left(t_{k}\right)\right\|\right),
$$

where the values of the constants $\kappa_{1}$ and $\kappa_{2}$ depend on the mobile environment, with $\kappa_{1}=0$ and $\kappa_{2}=30 \mathrm{~dB}$ being typical values in an urban setting [14], [19]. The parameter $\kappa_{1}$ captures the pilot signal strength, the antenna gains, and other constants that pertain to the signal propagation effects. Here, $\mathbf{r}\left(t_{k}\right)$ and $\mathbf{b}_{i}\left(t_{k}\right)$ are the position vectors at time $k$ for the MS and the $\mathrm{BS}_{i}$, respectively. The processes $\left\{W_{i}[k]\right\}, i \in \mathcal{C}$, are independent ${ }^{2}$, zero-mean, stationary Gaussian processes characterized by an autocorrelation function given by [13], [18]

$$
R_{W_{i}}(m)=\sigma_{W_{i}}^{2} \exp \left(\frac{-|m| d_{s}}{d_{0}}\right) .
$$

To eliminate the fast fading component $Z_{i}[k]$, the received signal strength $Y_{i}[k]$ is processed by an exponential smoothing window given by

$$
f_{\mathrm{av}}[k]=\frac{1}{d_{\mathrm{av}}} \exp \left(\frac{-k d_{s}}{d_{\mathrm{av}}}\right)=\frac{b^{k}}{d_{\mathrm{av}}}, \quad k \geq 0,
$$

where $b \triangleq \exp \left(-d_{s} / d_{\mathrm{av}}\right)$, and $d_{\mathrm{av}}$ determines the effective window size of the exponential averaging filter. Hence, the processed pilot signal strength from base station $\mathrm{BS}_{i}$ is obtained as follows:

$$
X_{i}[k]=f_{\mathrm{av}}[k] * Y_{i}[k], \quad k \geq 0,
$$

\footnotetext{
${ }^{1}$ The BS transmit signal strength is normalized to $0 \mathrm{~dB}$.

${ }^{2}$ If necessary, cross-correlation between received signal strengths can easily be incorporated into the model.
} 
where $*$ denotes discrete-time convolution. It can be shown [11] that $\left\{X_{i}[k]\right\}$ is a second-order autoregressive (AR) process.

\section{B. Handoff Analysis}

An important class of handoff algorithms operates on the basis of the relative processed signal strength between two candidate base stations $\mathrm{BS}_{i}$ and $\mathrm{BS}_{j}$ [9]-[11]. The relative processed signal strength between base stations $\mathrm{BS}_{i}$ and $\mathrm{BS}_{j}$ is denoted by $X[k] \triangleq X_{i}[k]-X_{j}[k]$. A handoff of the mobile user from base station $\mathrm{BS}_{i}$ to base station $\mathrm{BS}_{j}$ occurs when the relative processed signal strength falls below the value $-h_{j}$. Conversely, a handoff from base station $\mathrm{BS}_{j}$ to base station $\mathrm{BS}_{i}$ occurs when $X[k]$ exceeds the value $h_{i}$. The values $h_{i}$ and $h_{j}$ are known as the hysteresis levels associated with base stations $\mathrm{BS}_{i}$ and $\mathrm{BS}_{j}$, respectively.

In [11], handoff behavior is characterized in terms of the regions to which the relative signal strength is assigned at each value of the discrete-time parameter $k$. We define three assignment regions, which are intervals of the real-line:

$$
I \triangleq\left[h_{i}, \infty\right), J \triangleq\left(-\infty,-h_{j}\right], H \triangleq\left(-h_{j}, h_{i}\right) .
$$

The assignment regions at system initialization time are defined as $I_{0} \triangleq[0, \infty)$ and $J_{0} \triangleq(-\infty, 0)$.

For conventional handoff, the mobile will be assigned to base station $i$ if $X[k] \in I$ or if $X[l] \in I$ for some $l<k$ and $X[m] \in H$ for $l<m \leq k$. Let $X[k \backslash r]$ denote the sequence consisting of the $r$ most recent values of $\{X[k]\}$ up to and including time $k$, where $1 \leq r \leq k+1$ :

$$
X[k \backslash r] \triangleq(X[k-r+1], X[k-r+2], \cdots X[k]) .
$$

With respect to handoff behavior, a sequence $X[k \backslash r]$ can be represented equivalently by a string of length $k$ defined over the alphabet $\mathcal{A} \triangleq\left\{I_{0}, I, J_{0}, J, H\right\}$.

We shall use the notation $H^{r}$ to denote a string consisting of the symbol $H$ repeated $r$ times. When $k=0, X[k]$ falls in either region $I_{0}$ or region $J_{0}$. At any time $k \geq 1, X[k]$ falls in exactly one of the three assignment regions $I, J$, and $H$. Cell assignment of an MS along a given trajectory can be characterized as follows: for $k \geq 1$, if the MS is assigned to $\mathrm{BS}_{i}$ at time $k-1$, a handoff to cell $j$ occurs at time $k$ if and only if $X[k] \in J$. Conversely, if the MS is assigned to $\mathrm{BS}_{j}$ at time $k-1$, a handoff to cell $i$ occurs at time $k$ if and only if $X[k] \in I$.

Let $E_{i}[k]$ denote the event that the mobile is assigned to base station $\mathrm{BS}_{i}$ at time $k$. We refer to $E_{i}[k]$ as the cell $i$ assignment event at time $k$. We can express the assignment event, $E_{i}[k]$, as a disjoint union of more elementary events $^{3}$ [11]:

$$
E_{i}[k]=\left\{X[k \backslash k+1] \in I_{0} H^{k}\right\} \sqcup \bigsqcup_{r=1}^{k}\left\{X[k \backslash r] \in I H^{r-1}\right\} .
$$

Similarly, we can express the assignment event to base station $\mathrm{BS}_{j}$, denoted by $E_{j}[k]$, as in (7) by replacing $I_{0}$ and $I$ with $J_{0}$ and $J$, respectively. Let $E_{i j}[k]$ denote the event that a handoff

\footnotetext{
${ }^{3}$ When sets $\mathrm{A}$ and $\mathrm{B}$ are disjoint we denote their union by $A \sqcup B$ to emphasize this property.
}

from $\mathrm{BS}_{i}$ to $\mathrm{BS}_{j}$ occurs at time $k$. Then the handoff event can be expressed as follows:

$E_{i j}[k]=\left\{X[k \backslash k+1] \in I_{0} H^{k-1} J\right\} \sqcup \bigsqcup_{r=1}^{k}\left\{X[k \backslash r] \in I H^{r-2} J\right\}$.

In [11], a recursive procedure is developed to compute the assignment and handoff probabilities for hysteresis-based hard handoff algorithms. The recursive procedure is specified in terms of a sequence of bivariate functions defined below:

$$
\begin{aligned}
& g_{1}\left(x_{0}, x_{1}\right) \triangleq f_{1}\left(x_{0}, x_{1}\right), \\
& g_{2}\left(x_{1}, x_{2}\right) \triangleq \int_{I_{0}} g_{1}\left(x_{0}, x_{1}\right) f_{2}\left(x_{2} \mid x_{0}, x_{1}\right) d x_{0}, \\
& g_{k}\left(x_{k-1}, x_{k}\right) \triangleq \int_{I} f_{k-1}\left(x_{k-2}, x_{k-1}\right) f_{k}\left(x_{k} \mid x_{k-2}, x_{k-1}\right) d x_{k-2} \\
&+\int_{H} g_{k-1}\left(x_{k-2}, x_{k-1}\right) f_{k}\left(x_{k} \mid x_{k-2}, x_{k-1}\right) d x_{k-2},
\end{aligned}
$$

for $k \geq 3$. Here, $f_{k}\left(x_{k-1}, x_{k}\right)$ denotes the joint density of $\{X[k-1], X[k]\}$ and $f_{k}\left(x_{k} \mid x_{k-2}, x_{k-1}\right)$ is the conditional density of $X[k]$ given $\left\{X[k-1]=x_{k-1}\right\}$ and $\{X[k-2]=$ $\left.x_{k-2}\right\}$. Both densities are Gaussian. The bivariate functions $g_{k}$ are introduced as a mathematical device to simplify the calculation of handoff and assignment probabilities. The function $g_{1}\left(x_{0}, x_{1}\right)$ is simply the joint pdf of $X_{0}$ and $X_{1}$. The function $g_{2}\left(x_{1}, x_{2}\right)$ is the joint density of $X_{1}$ and $X_{2}$ such that $X_{0}$ falls in region $I_{0}$. For $k \geq 2, g_{k}\left(x_{k-1}, x_{k}\right)$ defines a joint density of $X_{k-1}$ and $X_{k}$ such that the path history of $X_{0}, \cdots, X_{k-2}$ does not result in a handoff from $\mathrm{BS}_{i}$ to $\mathrm{BS}_{j}$ at time $k-2$.

We introduce the notation

$$
p_{k}(\mathbf{S}) \triangleq \operatorname{Pr}\{X[k \backslash|\mathbf{S}|] \in \mathbf{S}\},
$$

where $\mathbf{S}$ is a string of symbols on the assignment alphabet $\mathcal{A}$ with length denoted by $|\mathbf{S}|$. The probability of assignment to $\mathrm{BS}_{i}$, defined by $P_{i}[k] \triangleq \operatorname{Pr}\left(E_{i}[k]\right)$, can be computed as ${ }^{4}$ :

$$
P_{i}[k]=p_{k}(I)+p_{k}(I H)+\iint_{H H} g_{k}\left(x_{k-1}, x_{k}\right) d x_{[k \backslash 2]},
$$

for $k \geq 2$, with the initial conditions

$$
P_{i}[1]=p_{1}(I)+p_{1}\left(I_{0} H\right) \text { and } P_{i}[0]=p_{0}\left(I_{0}\right) \text {. }
$$

Similarly, the handoff probability from $\mathrm{BS}_{i}$ to $\mathrm{BS}_{j}$, defined by $P_{i j}[k] \triangleq \operatorname{Pr}\left(E_{i j}[k]\right)$, can be expressed in terms of the functions $g_{k}$ as follows:

$$
P_{i j}[k]=p_{k}(I J)+\iint_{H J} g_{k}\left(x_{k-1}, x_{k}\right) d x_{[k \backslash 2]},
$$

for $k \geq 2$, with the initial condition $P_{i j}[1]=p_{1}\left(I_{0} J\right)$.

From the above handoff probabilities, the mean number of handoffs, $\bar{N}_{\text {ho }}$, that occur as the mobile moves along a given trajectory, is given by

$$
\bar{N}_{\text {ho }}=\sum_{k=1}^{K}\left(P_{i j}[k]+P_{j i}[k]\right),
$$

where $K$ is the total number of sampling intervals for a mobile moving along a given trajectory. In Sections IV and III, we will

\footnotetext{
${ }^{4}$ See Appendix A for an explanation of the compact notation used for integrals.
} 
present exact recursive algorithms for computing the outage probability and handoff interference, respectively, in terms of the functions $g_{k}$.

\section{HANDOFF INTERFERENCE}

\section{A. Definition of Handoff Interference}

As mentioned in the previous section, a handoff of the MS from base station $\mathrm{BS}_{i}$ to $\mathrm{BS}_{j}$ occurs when the relative processed signal strength $X[k]$ falls below the hysteresis level $-h_{j}$, and a handoff from $\mathrm{BS}_{j}$ to $\mathrm{BS}_{i}$ occurs when $X[k]$ exceeds the hysteresis level $h_{i}$. The mobile is assigned to base station $\mathrm{BS}_{i}$ at time $k$ if $X[k] \in I$ or if $X[l] \in I$ for some $l<k$ and $X[j] \in H$ for $l<j \leq k$.

The pilot signal strength received by an MS while performing a handoff between $\mathrm{BS}_{i}$ and $\mathrm{BS}_{j}$ can be expressed as

$$
C[k]=B[k]+G_{i}[k]+G_{j}[k],
$$

where

$$
\begin{aligned}
B[k] & =\max \left(Y_{i}[k], Y_{j}[k]\right), \\
G_{i}[k] & =1_{E_{i}[k]} \cdot[Y[k]]^{-}, G_{j}[k]=1_{E_{j}[k]} \cdot[-Y[k]]^{-},
\end{aligned}
$$

and $Y[k] \triangleq Y_{i}[k]-Y_{j}[k]$. Here, we use the notation $1_{A}$ to denote the indicator function on the set $A$ and $[x]^{-} \triangleq$ $\min \{0, x\}$. Also, $E_{i}[k]$ and $E_{j}[k]$ denote the events that the MS is assigned to $\mathrm{BS}_{i}$ and $\mathrm{BS}_{j}$, respectively (cf. (7)). Note that $G_{i}[k] \leq 0$ and $G_{j}[k] \leq 0$. Furthermore, at each time $k$, either $G_{i}[k]=0$ or $G_{j}[k]=0$ (or both).

We call $G_{i}[k]$ and $G_{j}[k]$ the handoff attenuations due to the use of the weaker signal during the assignment of the MS to $\mathrm{BS}_{i}$ and $\mathrm{BS}_{j}$, respectively. Fig. 1 illustrates signals $G_{i}[k]$ and $G_{j}[k]$ driven by the relative processed signal strength, $X[k]$, and a handoff algorithm with hysteresis levels $h_{i}=h_{j}=$ $5 \mathrm{~dB}$. From the plots of $G_{i}[k]$ and $G_{j}[k]$, we observe that the handoff algorithm forces the MS and the base stations to transmit more power than necessary to ensure a certain reception threshold.

In Fig. 1, the handoff attenuation $G_{i}[k]$ is non-zero during the assignment to $\mathrm{BS}_{i}$ within the intervals $\left(k_{1}, k_{2}\right),\left(k_{9}, k_{10}\right)$, etc., while $G_{j}[k]$ is non-zero during the assignment to $\mathrm{BS}_{j}$, within the intervals $\left(k_{3}, k_{4}\right),\left(k_{5}, k_{6}\right)$, and $\left(k_{7}, k_{8}\right)$. The overall attenuation is given by $G[k]=G_{i}[k]+G_{j}[k]$, which can be interpreted as the difference between the received signal strength from the current base station and the highest among the received signal strengths from the candidate base stations, i.e., $\mathrm{BS}_{i}$ and $\mathrm{BS}_{j}$.

The overall handoff attenuation can be interpreted in terms of power requirements at the MS and at the BS. We assume that the pilot signal strength transmitted by each BS maintains a constant power. However, the BS that is currently assigned to the MS adjusts its data transmission power (not the pilot signal) appropriately to maintain communications on the forward link. Similarly, the MS adjusts its data transmission power to maintain the reverse link. We assume that such power control is handled by the system and our handoff analysis is independent of the particular power control scheme that is used, since handoff depends only on the pilot signals, which are kept at constant power. If the MS were assigned to the best candidate base station (i.e., other than the base station to which it is currently assigned), $-G[k] \mathrm{dB}$ less data transmission signal strength would be necessary on both the forward and reverse links. However, at time $k$, the MS remains assigned to the current base station due to the hysteresis of the handoff algorithm. This implies that the MS has to use $-G[k] \mathrm{dB}$ more data transmission power than if it were assigned to the best candidate base station at time $k$, which results in more interference introduced into the system, due to the handoff algorithm. This motivates the concept of handoff interference (HI), to quantify this additional interference caused by the handoff algorithm. By choosing the handoff parameters appropriately, the handoff interference can be controlled, thus improving the system design.

We define the handoff interference (HI) by

$$
U[k] \triangleq U_{i}[k]+U_{j}[k],
$$

where $U_{i}[k]=-G_{i}[k]$ and $U_{j}[k]=-G_{j}[k]$; hence, $U[k]=$ $-G[k]$. Ideally, the handoff and power control strategies should be designed to keep the handoff interference as small as possible subject to a constraint on the signaling load due to handoffs. Alternatively, the signaling load could be minimized subject to a constraint on the handoff interference.

We denote the mean handoff interference at time $k$ by $\bar{U}[k] \triangleq E\{U[k]\}$. For a given trajectory, we define the handoff margin (HM) as the maximum value of the mean handoff interference experienced by the MS over the trajectory: $\hat{U} \triangleq \max _{1 \leq k \leq K}\{\bar{U}[k]\}$, where $K$ is the number of handoff decision instants along the trajectory of interest. We define the maximum interference point (MIP) as the point on the trajectory at which the handoff margin is achieved. The MIP can be viewed as a cell boundary indicator similar to the crossover point as defined in [9]. However, the MIP can be determined for any trajectory while the crossover point is defined only for the straight-line trajectory connecting two neighboring base stations.

Handoff interference and the derived HM and MIP metrics serve as performance measures for the handoff algorithm and can be used to dimension the handoff parameters to achieve the desired system performance tradeoffs. Handoff interference analysis can be extended to the multiple cell/user scenario by summing the contributions of the handoff interference from each MS in the network to the overall signal-to-interference plus noise ratio (SINR). In this way, handoff behavior taking into account the movement of mobile stations over multiple cells (cf. [20], [21]) could be incorporated directly into the evaluation of overall system capacity. We note that in [14], the system capacity for a CDMA network is evaluated without considering the mobility of the MS. A full treatment of system capacity analysis incorporating mobility via handoff interference analysis is beyond the scope of the present paper.

\section{B. Evaluation of Handoff Interference}

We now present a recursive procedure to compute the mean handoff interference, making use of the bivariate functions $g_{k}$, defined in Section II. The expected value of the handoff interference, $\bar{U}_{i}[k]$ at time $k$, corresponding to the communication 

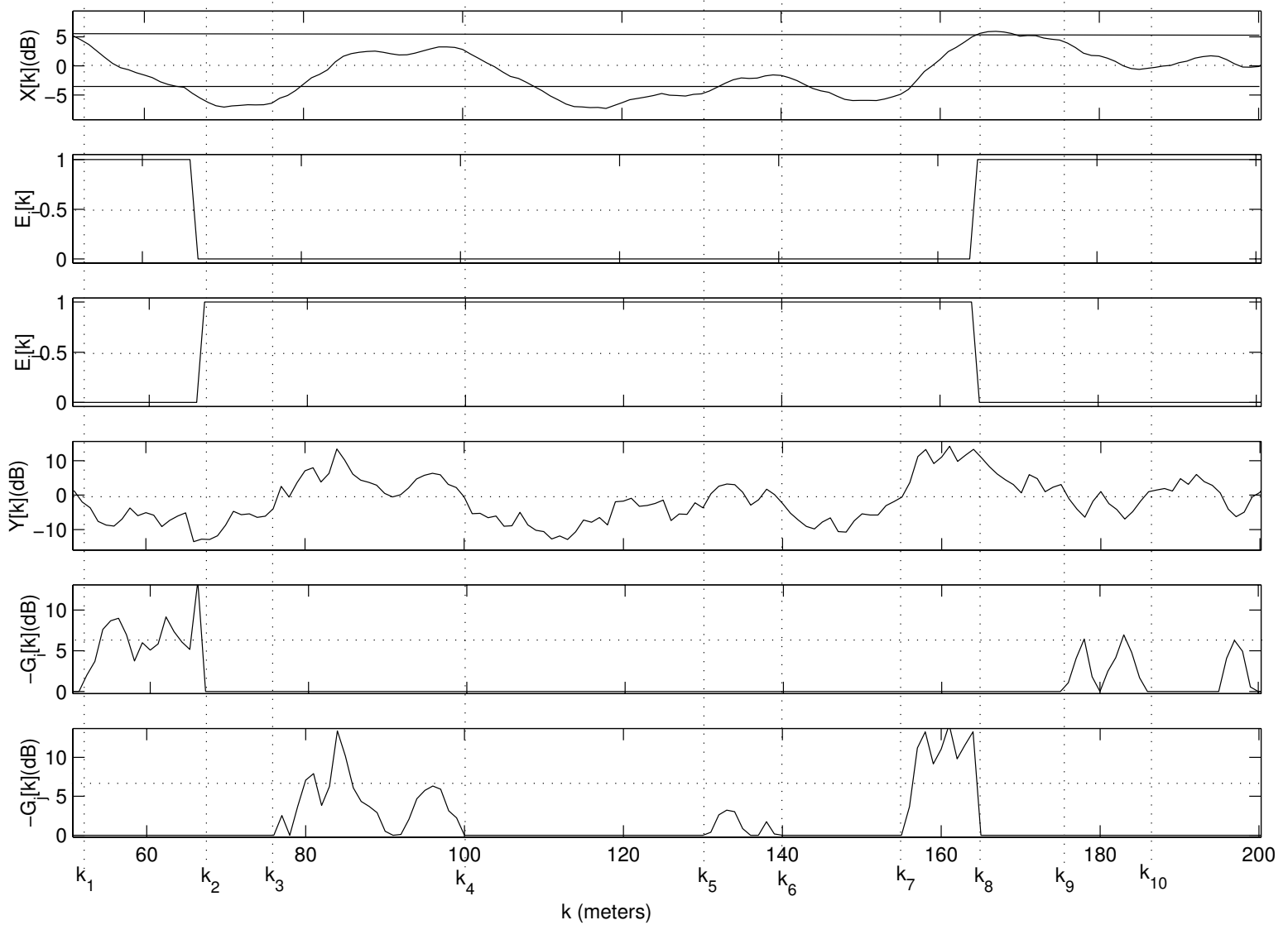

Fig. 1. Illustration of the handoff interference.

link with $\mathrm{BS}_{i}$ can be determined as follows:

$$
\begin{aligned}
\bar{U}_{i}[k] & =\iint_{\mathcal{R} I} \phi\left(x_{k-1}, x_{k}\right) \cdot f_{k-1, k}\left(x_{k-1}, x_{k}\right) d x_{[k \backslash 2]} \\
& +\iint_{I H} \phi\left(x_{k-1}, x_{k}\right) \cdot f_{k-1, k}\left(x_{k-1}, x_{k}\right) d x_{[k \backslash 2]} \\
& +\iint_{H H} \phi\left(x_{k-1}, x_{k}\right) \cdot g_{k}\left(x_{k-1}, x_{k}\right) d x_{[k \backslash 2]},
\end{aligned}
$$

where ${ }^{5}$

$$
\phi\left(x_{k-1}, x_{k}\right) \triangleq\left[2 d_{\mathrm{av}}\left(b x_{k-1}-x_{k}\right)\right]^{-}, \quad k \geq 2,
$$

with the initial conditions $\bar{U}_{i}[0]=0$ and

$$
\begin{aligned}
\bar{U}_{i}[1] & =\iint_{\mathcal{R} H} \phi\left(x_{0}, x_{1}\right) \cdot f_{0,1}\left(x_{0}, x_{1}\right) d x_{[1 \backslash 2]} \\
& +\iint_{I H} \phi\left(x_{0}, x_{1}\right) \cdot f_{0,1}\left(x_{0}, x_{1}\right) d x_{[1 \backslash 2]} .
\end{aligned}
$$

The mean HI, $\bar{U}_{j}[k]$, corresponding to $\mathrm{BS}_{j}$ can be calculated similarly.

\section{Outage Analysis}

\section{A. Outage Events}

An outage event can occur while the MS is assigned either to $\mathrm{BS}_{i}$ or $\mathrm{BS}_{j}$. Let $L_{i}[k]$ and $L_{j}[k]$ denote the events when

${ }^{5}$ The bivariate function $\phi\left(x_{k-1}, x_{k}\right)$ is related to $Y[k]$ by $\phi(X[k-$ $1], X[k])=2 d_{\mathrm{av}}[b X[k-1]-X[k]]^{-}=2[Y[k]]^{-}$. Based on (12), the relationship between $\phi(X[k-1], X[k])$ and $G_{i}[k]$ can be seen. A similar relationship holds between $\phi(X[k-1], X[k])$ and $G_{j}[k]$. the levels of the received signal strengths $Y_{i}[k]$ and $Y_{j}[k]$, respectively, fall below a certain threshold $T$, where $T$ is called the minimum received signal strength (MRSS), a quality-ofservice (QoS) parameter defined by the network provider:

$$
L_{i}[k] \triangleq\left\{Y_{i}[k]<T\right\} \text { and } L_{j}[k] \triangleq\left\{Y_{j}[k]<T\right\} .
$$

The outage event at time $k$ is defined by

$$
Q[k] \triangleq\left(E_{i}[k] \cap L_{i}[k]\right) \sqcup\left(E_{j}[k] \cap L_{j}[k]\right)=Q_{i}[k] \sqcup Q_{j}[k],
$$

where

$$
Q_{i}[k] \triangleq E_{i}[k] \cap L_{i}[k] \text { and } Q_{j}[k] \triangleq E_{j}[k] \cap L_{j}[k] .
$$

Here, $Q_{i}[k]$ denotes the outage event at time $k$ when the MS is assigned to cell $i$. Similarly, $Q_{j}[k]$ denotes the outage event at time $k$ when the MS is assigned to cell $j$.

The outage events $Q_{i}[k]$ and $Q_{j}[k]$, for $k \geq 1$, can be expressed as follows:

$$
\begin{aligned}
Q_{i}[k] & =\left\{X[k \backslash k+1] \in I_{0} H^{k} \cap L_{i}[k]\right\} \\
& \sqcup \bigsqcup_{r=1}^{k}\left\{X[k \backslash r] \in I H^{r-1} \cap L_{i}[k]\right\}, \\
Q_{j}[k] & =\left\{X[k \backslash k+1] \in J_{0} H^{k} \cap L_{j}[k]\right\} \\
& \sqcup \bigsqcup_{r=1}^{k}\left\{X[k \backslash r] \in J H^{r-1} \cap L_{j}[k]\right\} .
\end{aligned}
$$


For convenience, we introduce the notation

$$
o_{k}\left(\mathbf{S}, L_{i}\right) \triangleq \operatorname{Pr}\left\{X[k \backslash|\mathbf{S}|] \in \mathbf{S} \cap L_{i}[k]\right\},
$$

where $\mathbf{S}$ is a string of symbols on the assignment alphabet $\mathcal{A}$ with length denoted by $|\mathbf{S}|$. We define the outage probabilities in cells $i$ and $j$ by

$$
O_{i}[k] \triangleq \operatorname{Pr}\left\{Q_{i}[k]\right\} \text { and } O_{j}[k] \triangleq \operatorname{Pr}\left\{Q_{j}[k]\right\},
$$

respectively. Since the events on the right-hand sides of (15) and (16) are mutually exclusive, the outage probabilities in cells $i$ and $j$, and the overall outage probability can be expressed as follows.

Proposition 1: The overall outage probability is given by $O[k]=O_{i}[k]+O_{j}[k]$, where

$$
\begin{aligned}
& O_{i}[k]=o_{k}\left(I_{0} H^{k}, L_{i}\right)+\sum_{r=1}^{k} o_{k}\left(I H^{r-1}, L_{i}\right), \\
& O_{j}[k]=o_{k}\left(J_{0} H^{k}, L_{j}\right)+\sum_{r=1}^{k} o_{k}\left(J H^{r-1}, L_{j}\right) .
\end{aligned}
$$

\section{B. Evaluation of Outage Probability}

The probability $O_{i}[k]$ can be expressed in terms of the functions $g_{k}$ as follows ${ }^{6}$ :

$$
\begin{aligned}
O_{i}[k] & =o_{k}\left(I, L_{i}\right) \\
& +\iiint_{D_{i} I H} f_{k}\left(x_{k-1}, x_{k}\right) f_{i, k}\left(y_{i} \mid x_{k-1}, x_{k}\right) d x_{[k \backslash 2]} d y_{i} \\
& +\iiint_{D_{i} H^{2}} g_{k}\left(x_{k-1}, x_{k}\right) f_{i, k}\left(y_{i} \mid x_{k-1}, x_{k}\right) d x_{[k \backslash 2]} d y_{i},
\end{aligned}
$$

for $k \geq 2$, with the initial condition $O_{i}[1]=o_{1}\left(I_{0} H, L_{i}\right)+$ $o_{1}\left(I, L_{i}\right)$, where $D_{i}=(-\infty, T)$. The probability $o_{k}\left(I, L_{i}\right)$ is given by

$o_{k}\left(I, L_{i}\right)=\iiint_{D_{i} \mathcal{R} I} f_{k}\left(x_{k-1}, x_{k}\right) f_{i, k}\left(y_{i} \mid x_{k-1}, x_{k}\right) d x_{[k \backslash 2]} d y_{i}$,

for $k \geq 1$, where $\mathcal{R} \triangleq(-\infty, \infty)$, and $f_{i, k}\left(y_{i} \mid x_{k-1}, x_{k}\right)$ is the conditional density of $Y_{i}[k]$ given $X[k]$ and $X[k-1]$. An expression for this density function is given in Appendix $\mathrm{C}$. The probability $O_{j}[k]$, can be expressed similarly as in the above equation by replacing $i, I$, and $D_{i}$ with $j, J$, and $D_{j}$, respectively.

\section{TRAJECTORY CHARACTERIZATION FOR HANDOFF PERFORMANCE}

In this section, we develop a characterization of handoff performance over arbitrary mobile trajectories in a cellular network having a regular hexagonal cell pattern. Our approach can be extended to networks with other types of regular cell structure. Fig. 2 illustrates an example of a mobile trajectory inside a wireless network covered by hexagonal cells. In the hard handoff scheme, at a given location, only the two base stations closest to the mobile station are involved in the cell assignment process. The two base stations define a rhombus in which the major diagonal is a line segment joining the

\footnotetext{
${ }^{6}$ The equivalence between (19) and (17) is shown in Appendix B
}

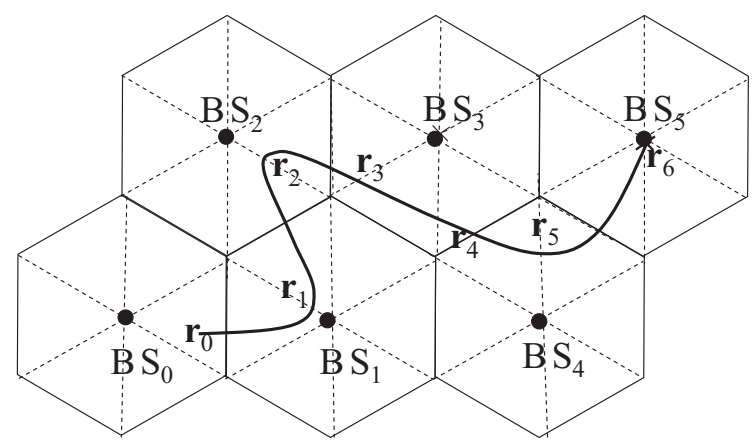

Fig. 2. A mobile trajectory in a cellular network.

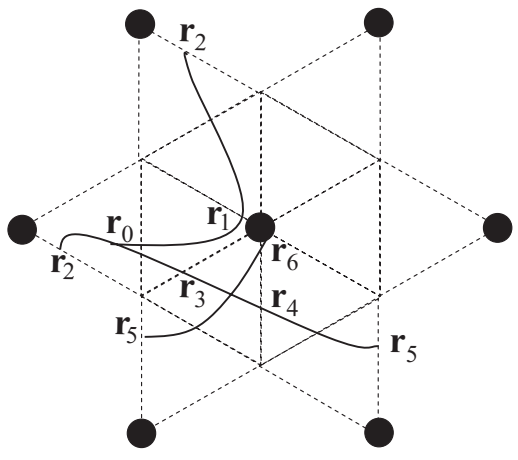

Fig. 3. Reduced geometric structure.

two base stations. We refer to this rhombus as a minimum geometric structure (MGS).

For the purposes of modeling and analyzing hard handoff performance, an arbitrary trajectory can be mapped into a single instance of an MGS. In order to accomplish this, we first introduce the concept of a reduced geometric structure (RGS). As shown in Fig. 3, an RGS is a star-shaped object consisting of a hexagonal cell at the center and six triangular regions subtending the edges of the hexagon. The mapping of the cellular network into a single MGS is performed in two steps:

1) Translation of all path segments into a single reduced geometric structure (RGS);

2) Rotation of all path segments within the RGS into a single MGS.

In the hexagonal cellular network, the RGS has the shape of a star, with a base station at the center and six neighboring base stations situated at the vertices of the star. The RGS may be viewed as consisting of six MGS's, one corresponding to each of the vertices.

The mapping of a mobile trajectory in a cellular network into an RGS is illustrated in Figs. 2 and 3. In Fig. 2, base station $\mathrm{BS}_{1}$ lies at the center of an RGS, that is chosen as the base RGS. The mobile trajectory begins at location $r_{0}$ in the RGS centered at $\mathrm{BS}_{1}$ and enters the RGS centered at $\mathrm{BS}_{3}$, at location $r_{2}$. Since the path segment $r_{0} r_{2}$ lies entirely within the base RGS, no translation is required. The path segment $r_{2} r_{5}$ lies within the RGS centered at $\mathrm{BS}_{3}$ and must be translated to the base RGS. This is done by translating the RGS centered at $\mathrm{BS}_{3}$ to the RGS centered at $\mathrm{BS}_{1}$. Fig. 3 shows the segments of the original trajectory that have been translated to the base RGS. For example, the path segment 


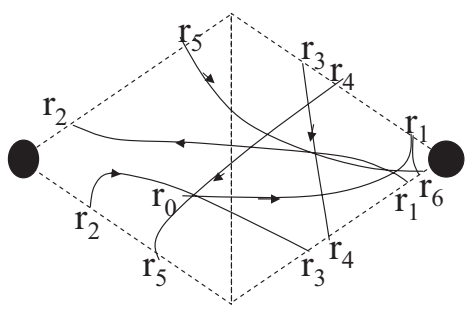

Fig. 4. Minimum geometric structure.

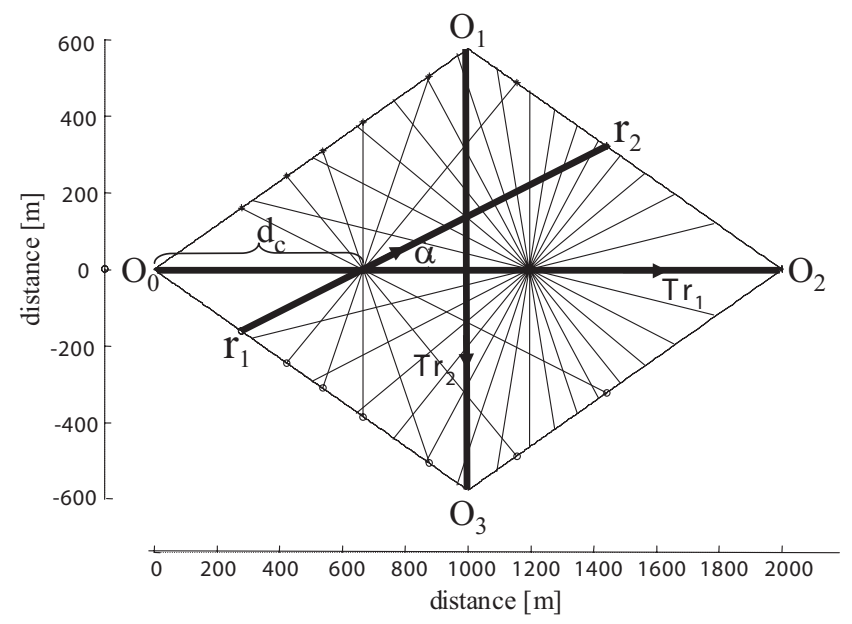

Fig. 5. Straight-line approximation trajectories within a minimum geometric structure.

$r_{2} r_{5}$, originally lying within the RGS centered at $\mathrm{BS}_{3}$ in Fig. 2 has been translated to its corresponding position within the base RGS in Fig. 3. Similarly, the path segment $r_{5} r_{6}$, originally lying within the RGS centered at $\mathrm{BS}_{4}$, has been translated to its corresponding position in the base RGS in Fig. 3. Thus, Fig. 3 shows a representation of the original mobile trajectory in the cellular network that has been mapped to a single base RGS via translation.

The trajectory segments shown in the base RGS of Fig. 3 can be further mapped into an MGS via rotations. The RGS in Fig. 3 consists of six rhombi corresponding to the vertices of the RGS. We choose the rhombus corresponding to the leftmost vertex of the RGS to serve as the base MGS. The remaining five MGS's are each rotated in turn to coincide with the base MGS. Within an MGS, a trajectory that passes from one boundary to another can be approximated by a line segment. As shown in Fig. 5, the segment $r_{1} r_{2}$ can be characterized uniquely by the crossing point located at the distance $d_{c}$ from $O_{0}$ and the angle $\alpha$, which it forms with $\left(\mathrm{O}_{0} \mathrm{O}_{2}\right)$. A set of line segments within the MGS can be generated by the parameter pair $\left(d_{c}, \alpha\right)$ over the range $\mathcal{F}=\left(0, d_{\max }\right) \times(0, \pi)$ using a particular step pair $\left(\delta_{d_{c}}, \delta_{\alpha}\right)$. The overall handoff performance within a cellular network can be characterized in terms of the mean number of handoffs along each segment in the set of generated line segments.

\section{NumericAl RESUlts AND Discussions}

In this section, we first present numerical results to validate the accuracy of our analytical technique for handoff interference. We then evaluate the outage probability and discuss

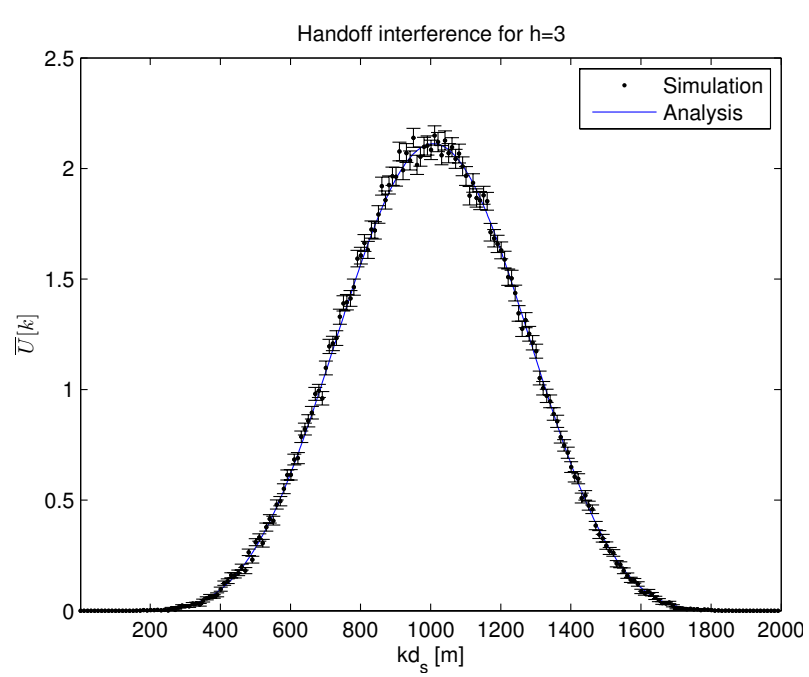

Fig. 6. Handoff interference along trajectory $\operatorname{Tr}_{1}$ for $h=3$.

the solution of a handoff design problem using the previous results. We set the main system parameters as follows: $D=$ $2000 \mathrm{~m}, \kappa_{1}=0 \mathrm{~dB}, \kappa_{2}=30 \mathrm{~dB}, \sigma_{W_{i}}=6 \mathrm{~dB}, d_{o}=20 \mathrm{~m}$, $d_{s}=1 \mathrm{~m}$. The averaging parameter $d_{\mathrm{av}}$ is set to $10 \mathrm{~m}$. The minimum received signal strength (MRSS) is set to be $-96 \mathrm{~dB}$, as in [22].

\section{A. Handoff Interference and Number of Handoffs}

In this subsection, we evaluate the handoff interference (HI) related metrics (e.g., mean HI, HM, and MIP) and the mean number of handoffs along the straight-line trajectory between two base stations, $\operatorname{Tr}_{1}$, within the minimum geometric structure of Fig. 5. The trajectory $\operatorname{Tr}_{1}$ is characterized by the pair $\left(d_{1}, \alpha_{1}\right)=(0,0)$. Fig. 6 shows the analysis of the mean handoff interference for $h=3 \mathrm{~dB}$, evaluated along the trajectory $\operatorname{Tr}_{1}$. The analytical curve is validated using computer simulation over 10,000 sample path iterations. The simulation results are shown in Fig. 6 along with 95\% confidence intervals. We observe that the mean handoff interference is initially zero near $\mathrm{BS}_{i}$ and it increases to a maximum of about $2.1 \mathrm{~dB}$, and then decreases to zero while the MS approaches the new base station, $\mathrm{BS}_{j}$. The maximum value of $2.1 \mathrm{~dB}$ represents the handoff margin, which occurs at approximately $1,010 \mathrm{~m}$ from the original base station. Thus, the maximum interference point for this trajectory is $1,010 \mathrm{~m}$.

In Fig. 7, the curve marked with triangles represents the handoff margin $\hat{U}$ versus the hysteresis level $h$ for the trajectory $\operatorname{Tr}_{1}$. On the same graph, we plot the mean number of handoffs. The mean number of handoffs decreases from 14 to 1 while the handoff margin increases from $1.2 \mathrm{~dB}$ to $5.4 \mathrm{~dB}$. Thus, to reduce the mean number of handoffs from 14 to 1 , the transmit power at the MS and base stations must be increased by about $4.2 \mathrm{~dB}$ or equivalently, a factor of 2.63 . The optimum hysteresis value for a given trajectory should be determined based on a cost function that trades off the handoff margin against the mean number of handoffs.

In Fig. 8, we compare the crossover point (COP) with the maximum interference point for trajectory $\operatorname{Tr}_{1}$. For $h=0 \mathrm{~dB}$, 


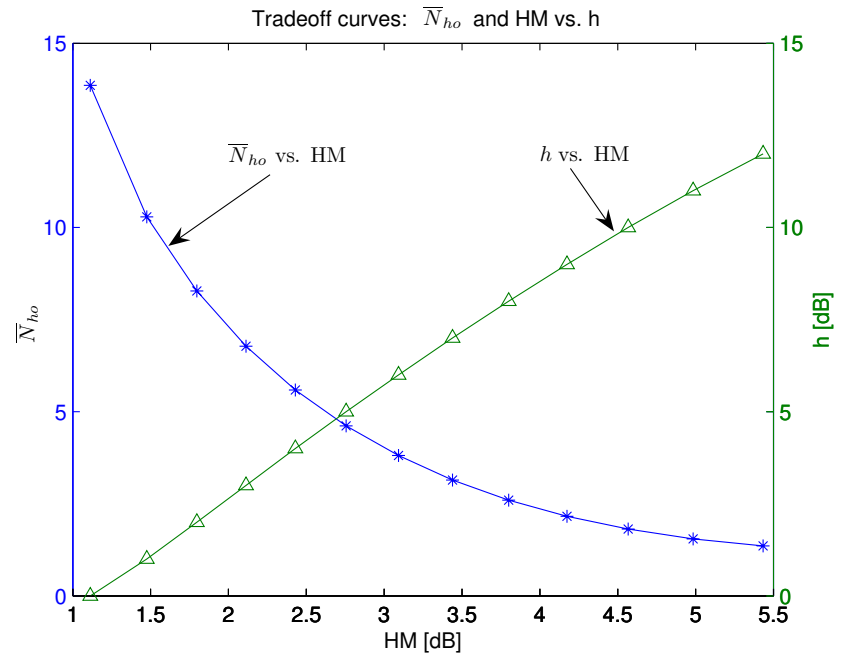

Fig. 7. HM and mean number of handoffs vs. hysteresis for $\operatorname{Tr}_{1}$.

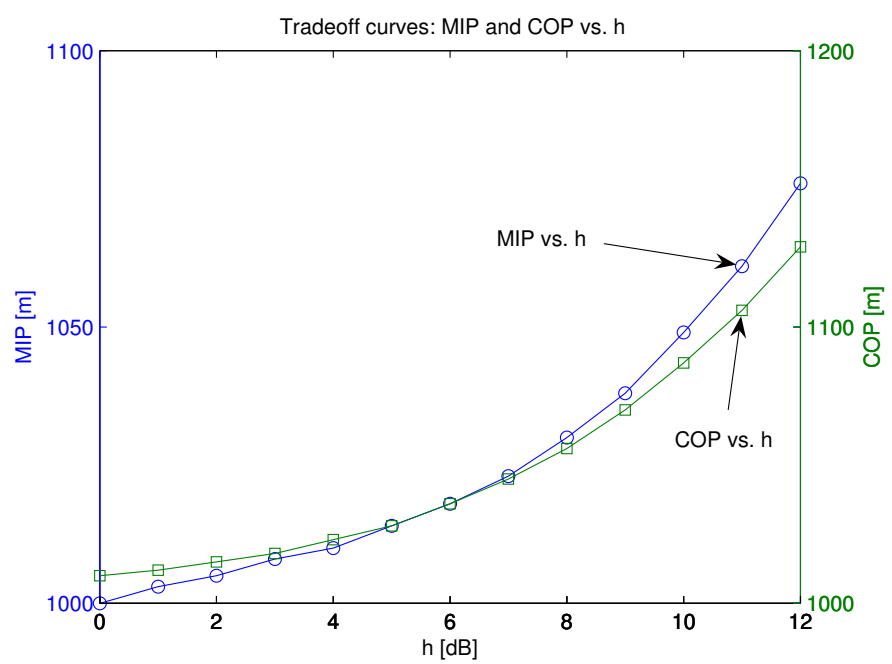

Fig. 8. MIP and crossover point vs. hysteresis for $\operatorname{Tr}_{1}$.

the MIP is exactly $1000 \mathrm{~m}$, i.e., at the midpoint of the trajectory, while the COP is slightly larger at $1,010 \mathrm{~m}$. The two curves cross at $h=6 \mathrm{~dB}$. Note that the MIP curve follows the same trend as the COP curve. On the other hand, the definition of COP makes sense only in the case of trajectory $\operatorname{Tr}_{1}$, whereas every trajectory has a well-defined MIP.

The mean number of handoffs for a cellular network with hexagonal structure can be characterized as a surface over the set $\mathcal{F}$ of straight-line trajectories in the minimum geometric structure discussed in Section V. Fig. 9 shows the mean handoff interference obtained over the set of straight-line trajectories within a minimum geometric structure when the hysteresis parameter $h$ is set to $1 \mathrm{~dB}$. The set $\mathcal{F}$ of line segments was generated using $\left(\delta_{d_{c}}, \delta_{\alpha}\right)=\left(100, \frac{\pi}{18}\right)$. In Fig. 9, one observes that the handoff margin surface is flat at the value of $2.2 \mathrm{~dB}$ for all segments intersecting the perpendicular trajectory, $\operatorname{Tr}_{2}$, represented by the pair $\left(d_{2}, \alpha_{2}\right)=(1000, \pi / 2)$. In particular, for all segments characterized by the distance crossing $d_{c}=1,000 \mathrm{~m}$, the handoff margin reaches the maximum value of the surface.

Fig. 10 shows the mean number of handoffs over the same set of straight-line trajectories as in Fig. 9 and the hysteresis

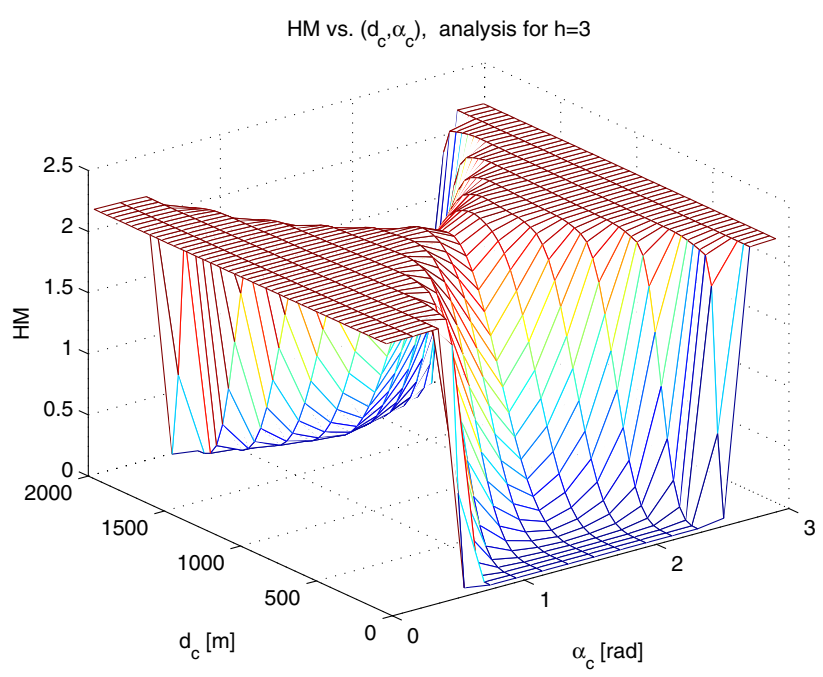

Fig. 9. Handoff margin, $h=3 \mathrm{~dB}$.

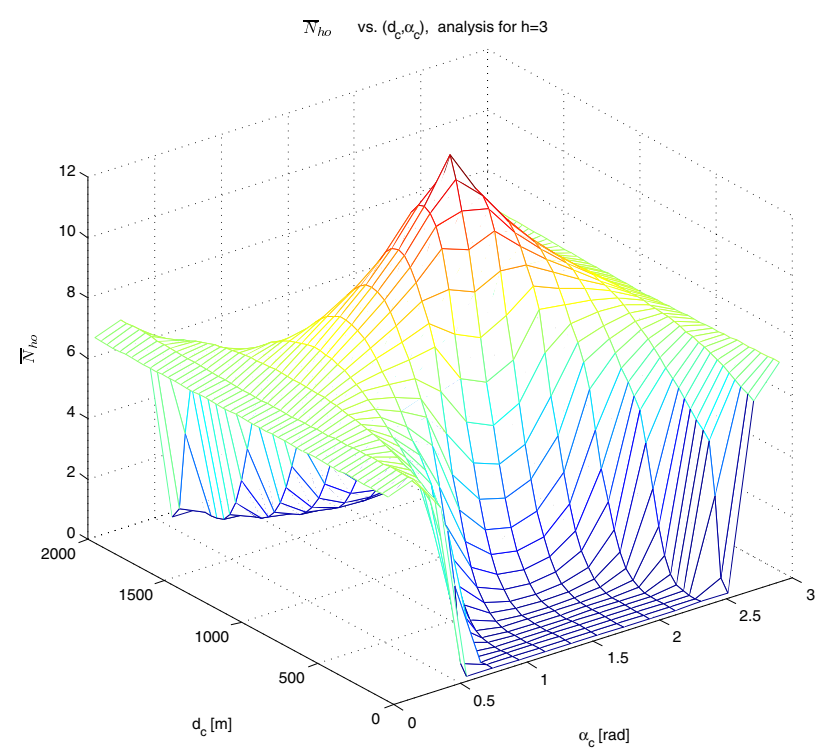

Fig. 10. Mean number of handoffs, $h=3 \mathrm{~dB}$.

parameter $h=1 \mathrm{~dB}$. One observes that the mean number of handoffs achieves a maximum when $d_{c}=1,000 \mathrm{~m}$ and $\alpha=$ $\pi / 2$, which corresponds to the trajectory $\operatorname{Tr}_{2}$ along the cell boundary between $\mathrm{BS}_{i}$ and $\mathrm{BS}_{j}$. For this particular trajectory, the mean number of handoffs is about 12 when $h=1 \mathrm{~dB}$. As $h$ is increased, the entire surface becomes lower, though not in a uniform manner.

\section{B. Outage Probability}

We now evaluate the outage probability and then discuss a specific design example. In Fig. 11, we observe that the analytical outage probability curves increase as the hysteresis level $h$ increases, which are verified by simulations with 10,000 iterations and $95 \%$ confidence intervals. The results are intuitive, because the larger the hysteresis level, the larger the handoff delay and the more likely an outage event occurs. We also observe that along a given trajectory between the two base stations $\mathrm{BS}_{i}$ and $\mathrm{BS}_{j}$, the outage probability first increases 


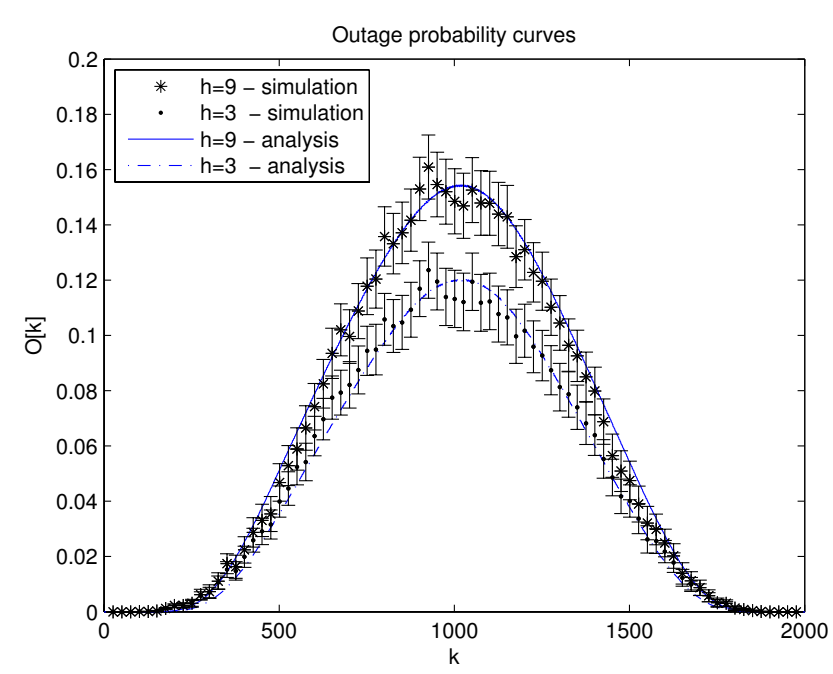

Fig. 11. Outage probability curves at $h=9 \mathrm{~dB}$ and $h=3 \mathrm{~dB}$.

until around the midpoint between the two base stations and then decreases from this midpoint. This is because the outage event happens more frequently around the midpoint between the two base stations, where the received signal strength is smaller.

\section{Handoff Design Example}

In the design and dimensioning of a cellular network, various optimization problems can be formulated with respect to the handoff performance metrics. For example, one may seek to determine the hysteresis level $h$ such that the outage probability satisfies a certain QoS requirement.

The following optimization problem incorporates handoff interference, outage probability, and mean number of handoffs into the optimal design of handoff parameters over a given mobile trajectory.

$$
\begin{aligned}
& \text { minimize } \mathrm{HC}=\mathrm{HM}+S_{t} \\
& \text { subject to: }\left\{\begin{array}{l}
\bar{N}_{\mathrm{ho}}(h) \leq \bar{N}_{0}, O_{k}\left(h, S_{t}\right) \leq O_{0}, \\
S_{\min } \leq S_{t} \leq S_{\max }, h \geq 0,
\end{array}\right.
\end{aligned}
$$

where HC denotes the handoff cost, HM is handoff margin, $S_{t}$ is the transmitted pilot signal strength, $\bar{N}_{\text {ho }}(h)$ is the mean number of handoffs, $O_{k}\left(h, S_{t}\right)$ is the average outage probability over the given trajectory, $O_{0}$ is a predefined threshold on the outage probability, $\bar{N}_{0}$ is a predefined threshold on the mean number of handoffs, $S_{\min }$ and $S_{\max }$ specify a predefined range of values for $S_{t}$. It is worthwhile to note that in the above constraints, $O_{k}$ is a function of both $h$ and $S_{t}$, while $\bar{N}_{\text {ho }}$ and $\mathrm{HM}$ are independent of $S_{t}$, since the handoff algorithm operates on the basis of the relative processed signal strength between two candidate base stations.

To solve the above optimization problem, we first determine the smallest value of $h$ such that $\bar{N}_{\text {ho }} \leq \bar{N}_{0}$. From the result of Fig. 7, i.e., the smaller the $h$, the smaller the HM, we see that such a value of $h$ will minimize HM. Then, we adjust the value of $S_{t}$ to get the smallest value of $S_{t}$ such that $O_{k}\left(h, S_{t}\right) \leq O_{0}$. Hence, the optimal values of $h$ and $S_{t}$ are obtained using two
TABLE I

RESULTS FOR THE OPTIMAL VALUES $h^{*}$ AND $S_{t}^{*}$

\begin{tabular}{|c|c||c|c|c|c|c|}
\hline $\bar{N}_{0}$ & $O_{0}$ & $h^{*}$ & $S_{t}^{*}$ & $\bar{N}_{\mathrm{ho}}\left(h^{*}\right)$ & $O_{k}\left(h^{*}, S_{t}^{*}\right)$ & $\mathrm{HC}(\mathrm{dB})$ \\
\hline \hline 8 & 0.05 & 2.5 & -0.5 & 7.5 & 0.0493 & 1.91 \\
\hline 5 & 0.05 & 5 & 0.5 & 4.6 & 0.0494 & 3.25 \\
\hline 3 & 0.05 & 7.5 & 1 & 2.9 & 0.047 & 4.62 \\
\hline
\end{tabular}

simple one-dimensional (1-D) searches for $h$ and $S_{t}$ such that

$$
\left\{\begin{array}{l}
h^{*}=\min \left\{h: \bar{N}_{\mathrm{ho}}(h) \leq \bar{N}_{0}\right\} \\
S_{t}^{*}=\min \left\{S_{t}: O_{k}\left(h^{*}, S_{t}\right) \leq O_{0}\right\} \\
S_{\min } \leq S_{t}^{*} \leq S_{\max }, h^{*} \geq 0
\end{array}\right.
$$

As a numerical example, we take the same system configuration as in the previous part of this section and optimize over the trajectory $\operatorname{Tr}_{1}$. The optimal values of $h$ and $S_{t}$ for different values of $\bar{N}_{0}$ and $O_{0}$ are shown in Table I.

\section{CONCLUSION}

We introduced a new performance measure to characterize handoff behavior called handoff interference. The handoff interference can be used as a metric for dimensioning the parameters of the handoff algorithm and to perform a comprehensive analysis of system capacity incorporating the mobility of the mobile station in multiple cell, multiple user scenarios. For a given set of user mobility patterns, the overall interference due to handoff in a multiple cell/user scenario can be evaluated by adding the handoff interference contributions from all mobile stations over time. We defined the handoff margin as the maximum of the mean handoff interference experienced by a mobile station along a given trajectory. The handoff parameters can be dimensioned to optimize handoff performance via a suitable tradeoff between the handoff margin and the mean number of handoffs. We defined the maximum interference point along a trajectory at which the handoff margin is achieved and showed that it generalizes the concept of crossover point, which was defined in earlier work as a measure of handoff delay for the special case of the straight-line trajectory connecting two base stations.

We presented exact recursive procedures to efficiently and accurately evaluate the handoff interference and the outage probability along a given trajectory for a class of hard handoff algorithms based on the relative processed pilot signal strength. We also described a methodology for characterizing a large class of trajectories in a cellular network with hexagonal cell structure. We showed that handoff performance could be characterized in terms of surfaces of mean number of handoffs and handoff margin over a set of straight-line approximation trajectories within a minimum geometric structure. These surfaces provide a convenient means of visualizing and computing the overall signaling load due to handoffs in the network. Finally, we discussed an example handoff design problem, which illustrates how to determine an optimal set of handoff parameters using results from our analysis. 


\section{APPENDIX}

\section{A. Notation for integrals}

We introduce the following compact notation for a multidimensional integral over the intervals $A_{k-n+1}, \ldots, A_{k}$, of the real line $(k \geq n-1)$ :

$$
\begin{aligned}
& \int_{A_{k-n+1} \ldots A_{k}} g\left(x_{k-n+1}, \ldots, x_{k}\right) d x_{[k \backslash n]} \triangleq \\
& \int_{A_{k-n+1}} \ldots \int_{A_{k}} g\left(x_{k-n+1}, \ldots, x_{k}\right) d x_{k} \ldots d x_{k-n+1} .
\end{aligned}
$$

Let $\mathbf{S}=A_{1} \ldots A_{n}$ be a string of symbols, each of which represents an interval of the real line. Then, the probability $p_{k}(\mathbf{S})$ defined in (9) can be expressed as $p_{k}(\mathbf{S})=$ $\int_{S} f_{k}\left(x_{k-n+1}, \ldots, x_{k}\right) d x_{[k \backslash n]}$.

\section{B. Derivation of (19):}

We derive (19) as follows:

$$
\begin{aligned}
O_{i}[k] & =o_{k}\left(I, L_{i}\right)+o_{k}\left(I H, L_{i}\right)+\int_{D_{i} I H^{2}} f_{k-1}\left(x_{k-2}, x_{k-1}\right) \\
& \cdot f_{k}\left(x_{k} \mid x_{k-2}, x_{k-1}\right) \cdot f_{i, k}\left(y_{i} \mid x_{k-1}, x_{k}\right) d x_{[k \backslash 3]} d y_{i} \\
& +\int_{D_{i} H^{3}} g_{k-1}\left(x_{k-2}, x_{k-1}\right) f_{k}\left(x_{k} \mid x_{k-2}, x_{k-1}\right) \\
& \cdot f_{i, k}\left(y_{i} \mid x_{k-1}, x_{k}\right) d x_{[k \backslash 3]} d y_{i} \\
& =o_{k}\left(I, L_{i}\right)+o_{k}\left(I H, L_{i}\right)+o_{k}\left(I H^{2}, L_{i}\right) \\
& +\int_{D_{i} I H^{3}} f_{k-2}\left(x_{k-3}, x_{k-2}\right) f_{k-1}\left(x_{k-1} \mid x_{k-3}, x_{k-2}\right) \\
& \cdot f_{k}\left(x_{k} \mid x_{k-2}, x_{k-1}\right) f_{i, k}\left(y_{i} \mid x_{k-1}, x_{k}\right) d x_{[k \backslash 4]} d y_{i} \\
& +\int_{D_{i} H^{4}} g_{k-2}\left(x_{k-3}, x_{k-2}\right) f_{k-1}\left(x_{k-1} \mid x_{k-3}, x_{k-2}\right) \\
& \cdot f_{k}\left(x_{k} \mid x_{k-2}, x_{k-1}\right) f_{i, k}\left(y_{i} \mid x_{k-1}, x_{k}\right) d x_{[k \backslash 4]} d y_{i}=\cdots \\
& =o_{k}\left(I, L_{i}\right)+o_{k}\left(I H, L_{i}\right)+o_{k}\left(I H^{2}, L_{i}\right)+\cdots \\
& +o_{k}\left(I H^{k-1}, L_{i}\right)+\int_{D_{i} H^{k}} g_{2}\left(x_{1}, x_{2}\right) f_{3}\left(x_{3} \mid x_{1}, x_{2}\right) \cdots \\
& \cdots f_{k}\left(x_{k} \mid x_{k-2}, x_{k-1}\right) f_{i, k}\left(y_{i} \mid x_{k-1}, x_{k}\right) d x_{[k \backslash k]} d y_{i} \\
& =\sum_{r=1}^{k} o_{k}\left(I H^{r-1}, L_{i}\right)+o_{k}\left(I_{0} H^{k}, L_{i}\right) .
\end{aligned}
$$

Note that the above derivation uses the second-order Markov property of the process $\{X[k]\}$.

\section{Derivation of $f_{i, k}\left(y_{i} \mid x_{k-1}, x_{k}\right)$}

Let $Y[k] \triangleq Y_{i}[k]-Y_{j}[k]$. From (5), we obtain

$$
Y[k]=d_{\mathrm{av}}(X[k]-b X[k-1]) .
$$

The conditional CDF is

$$
\begin{aligned}
F_{Y_{i}}\left(y \mid x_{k-1}, x_{k}\right) & =\operatorname{Pr}\left\{Y_{i}[k] \leq y \mid X[k-1]=x_{k-1}, X[k]=x_{k}\right\} \\
& =\operatorname{Pr}\left\{Y_{j}[k] \leq y-d_{\mathrm{av}}\left(x_{k}-b x_{k-1}\right)\right\} \\
& =F_{Y_{j}}\left(y+d_{\mathrm{av}}\left(b x_{k-1}-x_{k}\right)\right) .
\end{aligned}
$$

Taking the first derivative on both sides of the last equation, we obtain

$$
\begin{aligned}
& f_{Y_{i}}\left(y \mid x_{k-1}, x_{k}\right)=f_{Y_{j}}\left(y+d_{\mathrm{av}}\left(b x_{k-1}-x_{k}\right)\right) \\
& =\frac{1}{\sqrt{2 \pi} \sigma_{Y_{j}}} \exp \left\{-\frac{\left[y+d_{\mathrm{av}}\left(b x_{k-1}-x_{k}\right)-m_{Y_{j}}\right]^{2}}{2 \sigma_{Y_{j}}^{2}}\right\},
\end{aligned}
$$

where $m_{Y_{j}}$ and $\sigma_{Y_{j}}$ are the mean and standard deviation of $Y_{j}[k]$, respectively.

\section{REFERENCES}

[1] A. E. Leu and B. L. Mark, "Analysis of handoff interference along arbitrary trajectories in cellular networks," in Proc. IEEE WCNC '2004, May 2004, pp. 1969-1974.

[2] R. Zheng, W. Zhuang, and H. Jiang, "Scalable multiple description coding and distributed video streaming in 3G mobile communications," Wireless Commun. and Mobile Computing, vol. 5, no. 1, pp. 95-111, Feb. 2005.

[3] S. Frattasi, H. Fathi, F. H. P. Fitzek, R. Prasad, and M. D. Katz, "Defining 4G technology from the user's perspective," IEEE Network, vol. 20, no. 1, pp. 35-41, Jan.-Feb. 2006.

[4] C. Fu, F. Khendek, and R. Glitho, "Signaling for multimedia conferencing in 4G: the case of integrated 3G/MANETs," IEEE Commun. Mag., vol. 44, no. 8, pp. 90-99, Aug. 2006.

[5] S. Tang and W. Li, "Performance analysis of the $3 \mathrm{G}$ network with complementary WLANs," in Proc. IEEE Globecom'05, Nov. 2005, pp. $2637-2641$.

[6] H. Zhai, X. Chen, and Y. Fang, "A call admission and rate control scheme for multimedia support over IEEE 802.11 wireless LANs," Wireless Networks, vol. 12, no. 4, pp. 451-463, Aug. 2006.

[7] M. Shimakawa, D. Hole, and F. Tobagi, "Video-conferencing and data traffic over an IEEE 802.11g WLAN using DCF and EDCA," in Proc. IEEE ICC'05, May 2005, pp. 1324-1330.

[8] N. D. Tripathi, J. H. Reed, and H. F. VanLandingham, "Handoff in cellular systems," IEEE Personal Commun. Mag., vol. 5, no. 6, Dec. 1998

[9] R. Vijayan and J. M. Holtzman, "A model for analyzing handoff algorithms," IEEE Trans. Veh. Technol., vol. 42, no. 3, pp. 351-356, Aug. 1993.

[10] N. Zhang and J. M. Holtzman, "Analysis of handoff algorithms using both absolute and relative measurements," IEEE Trans. Veh. Technol., vol. 45, no. 1, pp. 174-179, Feb. 1996.

[11] A. E. Leu and B. L. Mark, "A discrete-time approach to analyze hard handoff performance in cellular networks," IEEE Trans. Wireless Commun., vol. 3, no. 5, pp. 1721-1733, Sept. 2004

[12] S. Ulukus and G. P. Pollini, "Handover delay in cellular wireless systems," in Proc. IEEE Int. Conf. on Comm (ICC)'98, June 1998, pp. $1370-1374$.

[13] M. Akar and U. Mitra, "Variations on optimal and suboptimal handoff control for wireless communication systems," IEEE J. Select. Areas Commun., vol. 19, no. 6, pp. 1173-1185, June 2001.

[14] A. J. Viterbi, A. M. Viterbi, K. S. Gilhousen, and E. Zehavi, "Soft handoff extends CDMA cell coverage and increases reverse link capacity," IEEE J. Select. Areas Commun., vol. 12, no. 8, pp. 1281-1288, Oct. 1994

[15] K. M. Rege, S. Nanda, C. F. Weaver, and W. C. Peng, "Fade margins for soft and hard handoffs," ACM J. Wireless Networks, vol. 2, no. 4, pp. 277-288, Dec. 1996.

[16] C. W. Sung, "Analysis of fade margins for soft and hard handoffs in cellular systems," IEEE Trans. Wireless Commun., vol. 2, no. 3, pp. 431-435, May 2003.

[17] A. E. Leu and B. L. Mark, "Discrete-time level-crossing analysis of soft handoff performance in cellular networks," IEEE Trans. Inform. Theory, vol. 52, no. 7, pp. 3283-2390, July 2006.

[18] M. Gudmundson, "Correlation model for shadowing fading in mobile radio systems," Electron. Lett., vol. 27, no. 23, pp. 2145-2146, Nov. 1991.

[19] W. C. Y. Lee, Mobile Communications Design Fundamentals, 2nd. ed. New York: Wiley, 1993.

[20] M. M. Zonooni and P. Dassanayake, "User mobility modeling and characterization of mobility patterns," IEEE J. Select. Areas Commun., vol. 15, no. 7, pp. 1239-1252, Sept. 1997.

[21] H. Kobayashi, S.-Z. Yu, and B. L. Mark, "An integrated mobility and traffic model for resource allocation in wireless networks," in Proc. 3rd ACM Int. Workshop on Wireless Mobile Multimedia, Boston, MA, Aug. 2000, pp. 39-47.

[22] R. Suleesathira and S. Kunarak, "Neural Network Handoff Algorithm in a Joint Terrestrial-HAPS Cellular System," ECTI Trans. Electrical Eng. Electron., and Commun., vol. 3, no. 2, pp. 164-174, Aug. 2005. 


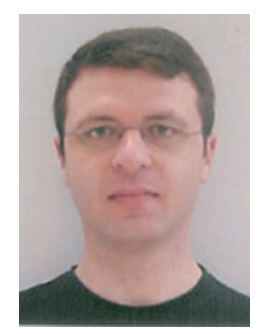

Alexe E. Leu (S'97, M'06) received the B.S. and M.S. degrees in Electronics and Telecommunications from Politechnica University of Bucharest, Romania, in 1995 and 1996, respectively and the Ph.D. degree Electrical and Computer Engineering from George Mason University, Fairfax, VA in 2003.

From 1999 to 2001, he was a Design Engineer in the R\&D Department at LCC International Inc, McLean, VA. Since 2003, he has been a Senior Research Engineer at Shared Spectrum Company, Vienna, VA. His research interests are in cognitive radio; design, modeling, and performance evaluation of wireless network architectures and protocols; integrating spatial and temporal processing for mobile networks; cross-layer design for wireless networking; and statistical modeling of wireless channels.

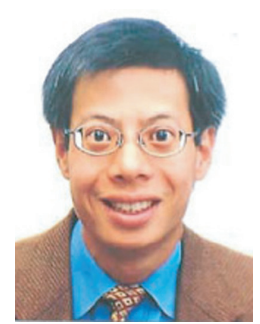

Brian L. Mark (M'91) received the B.A.Sc. degree in Computer Engineering with an option in Mathematics from the University of Waterloo, Canada, in 1991 and the Ph.D. in Electrical Engineering from Princeton University, Princeton, NJ, in 1995.

$\mathrm{He}$ was a Research Staff Member at the C\&C Research Laboratories, NEC USA, from 1995 to 1999. In 1999, he was on part-time leave from NEC as a visiting researcher at Ecole Nationale Supérieure des Télécommunications in Paris, France. In 2000, he joined the Dept. of Electrical and Computer Engineering at George Mason University, where he is currently an Associate Professor. His research interests lie in the design, modeling, and analysis of communication systems, computer systems, and communication networks. He was co-recipient of the best conference paper award for IEEE Infocom'97. He received a National Science Foundation CAREER Award in 2002. Currently, he is serving as an Associate Editor for IEEE TRANSACTIONS ON VEHICULAR TECHNOLOGY.

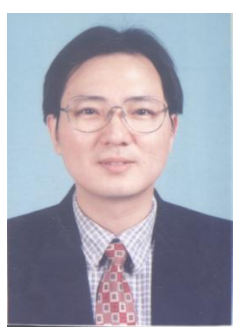

Shensheng Tang (SM'07) received his B.S. degree from Tianjin University, Tianjin, China, and M.S. degree from China Academy of Telecommunications Technology (CATT), Beijing, China, both in Electronic Engineering. He received his Ph.D. in Electrical Engineering from University of Toledo, Ohio. He has over 8 years of industrial experience in the field of information technology, which involves the product development of electronic measurement instruments and mobile communication equipments as well as R\&D of the 3G TD-SCDMA standard. During his time in industry, he has served as hardware engineer, system engineer and project manager, respectively. Currently, he is a research fellow in the Department of Electrical and Computer Engineering at George Mason University. His research interests focus on wireless networking and mobile computing, modeling and performance evaluation, digital signal processing, and network security. 